\title{
UDC: 577.3.01; 577.38 SOME EXACT AND NUMERICAL SOLUTION OF THE PROBLEM OF KOLMOGOROV-FISHER TYPE BIOLOGICAL POPULATION TASK WITH DOUBLE NONLINEAR DIFFUSION
}

\author{
Dildora Muhamediyeva ${ }^{1}$ \\ ${ }^{I}$ Tashkent University of information technologies named after Muhammad al-Khwarizmi Scientific and innovation \\ center of information and communication technologies, Tashkent, Uzbekistan
}

\begin{abstract}
In this paper, we study the global solvability of a task of Kolmogorov-Fisher type biological population task with double nonlinear diffusion and qualitative properties of the solution of the task based on the self-similar analysis.
\end{abstract}

Keywords: double nonlinearity, cross-diffusion, biological population, parabolic system of quasilinear equations, convective transport, numerical solution, iterative process, self-similar solutions.

\section{INTRODUCTION}

Population model are studied for a long time, first such work was performed by Fisher, Gause, and mathematical justification was performed by Kolmogorov, Petrovsky (KPP) and Piskunov (1937) in famous work (see. [8] and references therein). They were interested in the behavior of the velocity wave solutions and the resulting estimate of the speed of wave propagation. Then there were appear other models of the population. In recent years, were studied intensively nonlinear model taking into account diffusion and revealed new properties of the finite speed of propagation of diffusive waves (see [3] and the literature cited therein). We have proposed a model, which is described by a nonlinear system of competing species. Identified new properties such as the finite speed of propagation, localization outbreaks in a specific area. In particular, in the critical case the rate type KPP generalizes their result.

Consider in the field $Q=\{(t, x): 0<t, x \in R\}$ class of parabolic systems of two quasilinear equations of reactiondiffusion with double nonlinear diffusion [1-9]

$$
\left\{\begin{array}{l}
\frac{\partial u_{1}}{\partial t}=\operatorname{div}\left(D_{1} u_{2}^{m_{1}-1}\left|\nabla u_{1}\right|^{p-2} \nabla u_{1}\right)+f_{\beta_{1}}\left(u_{1}\right) \\
\frac{\partial u_{2}}{\partial t}=\operatorname{div}\left(D_{2} u_{1}^{m_{2}-1}\left|\nabla u_{2}\right|^{p-2} \nabla u_{2}\right)+f_{\beta_{2}}\left(u_{2}\right)
\end{array}\right.
$$

$\left.u_{1}\right|_{t=0}=u_{10}(x),\left.u_{2}\right|_{t=0}=u_{20}(x)$

which describes the process of Kolmogorov-Fisher type biological population in a nonlinear two-component environment at

$$
\begin{aligned}
& \left.f_{\beta_{1}}\left(u_{1}\right)=k_{1} t\right) u_{1}\left(1-u_{1}^{\beta_{1}}\right), \\
& f_{\beta_{2}}\left(u_{2}\right)=k_{2} u_{2}\left(1-u_{2}^{\beta_{2}}\right), \quad \text { where } \quad k_{1}=1 / \beta_{1}, \\
& k_{2}=1 / \beta_{2} \quad[10] \text { and } \\
& \left\{\begin{array}{l}
\frac{\partial u_{1}}{\partial t}=\frac{\partial}{\partial x}\left(D_{1} u_{2}^{m_{1}-1}\left|\frac{\partial u_{1}}{\partial x}\right|^{p-2} \frac{\partial u_{1}}{\partial x}\right)+k_{1} u_{1}\left(1-u_{1}^{\beta_{1}}\right) \\
\frac{\partial u_{2}}{\partial t}=\frac{\partial}{\partial x}\left(D_{2} u_{1}^{m_{2}-1}\left|\frac{\partial u_{2}}{\partial x}\right|^{p-2} \frac{\partial u_{2}}{\partial x}\right)+k_{2} u_{2}\left(1-u_{2}^{\beta_{2}}\right)
\end{array}\right.
\end{aligned}
$$

Diffusion coefficient of which is equal to $D_{1} u_{2}^{m_{1}-1}\left|\frac{\partial u_{1}}{\partial x}\right|^{p-2}$, $D_{2} u_{1}^{m_{2}-1}\left|\frac{\partial u_{2}}{\partial x}\right|^{p-2}$, where $m_{1}, m_{2}, p, \beta_{1}, \beta_{2}$ - positive real numbers, $u_{1}=u_{1}(t, x) \geq 0, \quad u_{2}=u_{2}(t, x) \geq 0$ $\begin{array}{lll}\text { desired } & \text { solution. } & \beta_{i} \rightarrow 0\end{array}$

$$
\begin{aligned}
& \lim _{\beta_{i} \rightarrow 0} f_{\beta_{i}}\left(u_{i}\right)=u_{i} \lim _{\beta_{i} \rightarrow 0}\left[\frac{1}{\beta_{i}}\left(1-u^{\beta_{i}}\right)\right]=\bar{f}\left(u_{i}\right), \\
& \bar{f}\left(u_{i}\right)=-u_{i} \ln u_{i}, \quad i=1,2 \quad[10] .
\end{aligned}
$$

Below we investigate the qualitative properties of the considered problem by constructing self-similar system of equations for (1).

\section{SELF-SIMILAR SYSTEM OF EQUATIONS}

Self-similar system of equations we will construct by the method of nonlinear splitting [1]. 
Substitution in (1)

$$
u_{1}(t, x)=e^{k_{1} t} v_{1}(t, \mathrm{x}), \quad u_{2}(t, x)=e^{k_{2} t} v_{2}(t, \mathrm{x}),
$$

Lead (1) to the form:

$$
\left\{\begin{array}{l}
\frac{\partial v_{1}}{\partial \tau_{1}}=\frac{\partial}{\partial x}\left(D_{1} v_{2}^{m_{1}-1}\left|\frac{\partial v_{1}}{\partial x}\right|^{p-2} \frac{\partial v_{1}}{\partial x}\right)+k_{1} e^{\left[\left(\beta_{1}-\mathrm{p}+2\right) \mathrm{k}_{1}-\left(m_{1}-1\right) k_{2}\right] t} v_{1}^{\beta_{1}+1} \\
\frac{\partial v_{2}}{\partial \tau_{2}}=\frac{\partial}{\partial x}\left(D_{2} v_{1}^{m_{2}-1}\left|\frac{\partial v_{2}}{\partial x}\right|^{p-2} \frac{\partial v_{2}}{\partial x}\right)+k_{2} e^{\left[\left(\beta_{2}-\mathrm{p}+2\right) \mathrm{k}_{2}-\left(m_{2}-1\right) k_{1}\right] t} v_{2}^{\beta_{2}+1}
\end{array}\right.
$$

$\left.v_{1}\right|_{t=0}=v_{10}(\eta),\left.\quad v_{2}\right|_{t=0}=v_{20}(\eta)$.

Choosing

$$
\tau=\frac{e^{\left[\left(m_{1}-1\right) k_{2}+(p-2) k_{1}\right] t}}{(p-2) k_{1}+\left(m_{1}-1\right) k_{2}}=\frac{e^{\left[\left(m_{1}-1\right) k_{1}+(p-2) k_{2}\right] t}}{(p-2) k_{2}+\left(m_{2}-1\right) k_{1}} ;,
$$

obtain the following system of equations:

$$
\left\{\begin{array}{l}
\frac{\partial v_{1}}{\partial \tau}=\frac{\partial}{\partial x}\left(D_{1} v_{2}^{m_{1}-1}\left|\frac{\partial v_{1}}{\partial x}\right|^{p-2} \frac{\partial v_{1}}{\partial x}\right)-a_{1} \tau^{b_{1}} v_{1}^{\beta_{1}+1} \\
\frac{\partial v_{2}}{\partial \tau}=\frac{\partial}{\partial x}\left(D_{2} v_{1}^{m_{2}-1}\left|\frac{\partial v_{2}}{\partial x}\right|^{p-2} \frac{\partial v_{2}}{\partial x}\right)-a_{2} \tau^{b_{2}} v_{2}^{\beta_{2}+1}
\end{array}\right.
$$

Where

$$
a_{1}(\mathrm{t})=k_{1}(\mathrm{t}) \cdot\left[(\mathrm{p}-2) \mathrm{k}_{1}+\left(m_{1}-1\right) k_{2}\right]^{b_{1}}
$$

And $\tau=\tau(t)$ is chosen as

$$
\begin{aligned}
& \tau(\tau)=\int_{0}^{\tau} v_{1}^{-(p-2)}(t) v_{2}^{-\left(m_{1}-1\right)}(t) d t=\int_{0}^{\tau} v_{2}^{-(p-2)}(t) v_{1}^{-\left(m_{2}-1\right)} d t= \\
& = \begin{cases}\frac{1}{1-\left[\alpha_{1}(\mathrm{p}-2)+\alpha_{2}\left(\mathrm{~m}_{1}-1\right)\right]}(\mathrm{T}+\tau)^{-\left[\alpha_{1}(\mathrm{p}-2)+\alpha_{2}\left(\mathrm{~m}_{1}-1\right)\right]+1}, & \text { if } 1-\left[\alpha_{1}(\mathrm{p}-2)+\alpha_{2}\left(\mathrm{~m}_{1}-1\right)\right] \neq 0, \\
\ln (T+\tau), & \text { if } 1-\left[\alpha_{1}(\mathrm{p}-2)+\alpha_{2}\left(\mathrm{~m}_{1}-1\right)\right]=0,\end{cases}
\end{aligned}
$$

With the aim of obtaining self-similar system for the system of equations (3) we find first the solution of a system of ordinary differential equations

$$
\left\{\begin{array}{l}
\frac{d \bar{\nu}_{1}}{d \tau}=-a_{1} \tau^{b_{1}} \bar{v}_{1}^{\beta_{1}+1}, \\
\frac{d \bar{\nu}_{2}}{d \tau}=-a_{2} \tau^{b_{2}} \bar{v}_{2}^{\beta_{2}+1}
\end{array}\right.
$$

In the form

$\overline{v_{1}}(\tau)=\tau^{-\alpha_{1}}, \overline{v_{2}}(\tau)=\tau^{-\alpha_{2}}$,

Where

$$
\alpha_{1}=\frac{b_{1}+1}{\beta_{1}}, \quad \alpha_{2}=\frac{b_{2}+1}{\beta_{2}} .
$$

Then the solution of system (3) is sought in the form

$$
v_{1}(t, x)=\bar{v}_{1}(t) w_{1}(\tau, x), v_{2}(t, x)=\bar{v}_{2}(t) w_{2}(\tau, x)
$$

if $\alpha_{1}(\mathrm{p}-2)+\alpha_{2}\left(\mathrm{~m}_{1}-1\right)=\alpha_{2}(\mathrm{p}-2)+\alpha_{1}\left(\mathrm{~m}_{2}-1\right)$.

Then for $w_{i}(\tau, x), i=1,2$ we get the system of equations 
$\left\{\begin{array}{l}\frac{\partial w_{1}}{\partial \tau}=\frac{\partial}{\partial x}\left(D_{1} w_{2}^{m_{1}-1}\left|\frac{\partial w_{1}}{\partial x}\right|^{p-2} \frac{\partial w_{1}}{\partial x}\right)+\psi_{1}\left(w_{1}^{\beta_{1}+1}-w_{1}\right) \\ \frac{\partial w_{2}}{\partial \tau}=\frac{\partial}{\partial x}\left(D_{2} w_{1}^{m_{2}-1}\left|\frac{\partial w_{2}}{\partial x}\right|^{p-2} \frac{\partial w_{2}}{\partial x}\right)+\psi_{2}\left(w_{2}^{\beta_{2}+1}-w_{2}\right)\end{array}\right.$

Where

$\psi_{i}=\frac{1}{\left(1-\left[\alpha_{i}(\mathrm{p}-2)+\alpha_{3-i}\left(\mathrm{~m}_{i}-1\right)\right]\right) \tau}, \quad i=1,2$

Consider the self-similar solution of the system (5) of the form

$$
w_{1}(t, x)=f_{1}(\xi), w_{2}(t, x)=f_{2}(\xi), \xi=|x| /\left(T+\tau^{T}\right.
$$

Then substituting (7) into (5) with respect to $f_{1}(\xi), f_{2}(\xi)$ obtain the following system of nonlinear degenerate self-similar equations:

$\left\{\begin{array}{l}\frac{d}{d \xi}\left(f_{2}^{m_{1}-1}\left|\frac{d f_{1}}{d \xi}\right|^{p-2} \frac{d f_{1}}{d \xi}\right)+\frac{\xi}{p} \frac{d f_{1}}{d \xi}+\theta_{1}\left(f_{1}-f_{1}^{\beta_{1}+1}\right)=0 \\ \frac{d}{d \xi}\left(f_{1}^{m_{2}-1}\left|\frac{d f_{2}}{d \xi}\right|^{p-2} \frac{d f_{2}}{d \xi}\right)+\frac{\xi}{p} \frac{d f_{2}}{d \xi}+\theta_{2}\left(f_{2}-f_{2}^{\beta_{2}+1}\right)=0\end{array}\right.$

Where

$$
\theta_{i}=\frac{1}{\left(1-\left[\alpha_{i}(\mathrm{p}-2)+\alpha_{3-i}\left(\mathrm{~m}_{i}-1\right)\right]\right) \tau}
$$

Let us build an upper solution for system (8).

\section{CONSTRUCTION OF UPPER SOLUTIONS} If

$$
\beta_{i}=\left[(\mathrm{p}-2)^{2}-\left(m_{1}-1\right)\left(m_{2}-1\right)\right] /\left[(\mathrm{p}-1)\left(\mathrm{p}-\left(\mathrm{m}_{i}+1\right)\right]\right.
$$

$$
p>2+\sqrt{\left(m_{1}-1\right)\left(m_{2}-1\right)}, i=1,2 \text {, equation (8) }
$$

has a local solution
$\bar{f}_{1}(\xi)=A\left(a-\xi^{\gamma}\right)_{+}^{{ }^{n_{1}}}, \bar{f}_{2}(\xi)=B\left(a-\xi^{\gamma}\right)_{+}^{{ }^{n_{2}}}$,

Where

$(b)_{+}=\max (0, b), \gamma=p /(p-1)$,

$n_{1}=\frac{(p-1)\left(p-\left(m_{1}+1\right)\right.}{(p-2)^{2}-\left(m_{1}-1\right)\left(m_{2}-1\right)}$

$n_{2}=\frac{(p-1)\left(p-\left(m_{2}+1\right)\right.}{(p-2)^{2}-\left(m_{1}-1\right)\left(m_{2}-1\right)}$.

Then in the field Q according to the comparison principle of solutions $[1,8]$ we have

Theorem 1: Let $\quad u_{i}(0, x) \leq u_{i \pm}(0, x), x \in R$.

Then for the solution of the problem (1) in the domain $\mathrm{Q}$ we have the estimate

$$
\begin{gathered}
u_{1}(t, x) \leq u_{1+}(t, x)=e^{k_{1} t} \tau^{-\alpha_{1}} \bar{f}_{1}(\xi) \\
u_{2}(t, x) \leq u_{2+}(t, x)=e^{k_{2} t} \tau^{-\alpha_{2}} \bar{f}_{2}(\xi) \\
\xi=|x| / \tau^{1 / p}
\end{gathered}
$$

where $\bar{f}_{1}(\xi), \bar{f}_{2}(\xi) u \tau(t)$ - certain of the above functions.

Similarly, [9] permanent $\boldsymbol{a}$ can be found from the condition

$$
\int_{-\infty}^{\infty} E_{P_{1}}(x, \tau) d x=P_{1}, \int_{-\infty}^{\infty} E_{P_{2}}(x, \tau) d x=P_{2}
$$

Where

$$
\begin{gathered}
E_{P_{1}}(x, \tau)=\tau^{-\frac{1}{\mu_{1}}}\left(a-\xi_{1}^{\gamma}\right)_{+}^{n_{1}}, E_{P_{2}}(x, \tau)=\tau^{-\frac{1}{\mu_{2}}}\left(a-\xi_{2}^{\gamma}\right)_{+}^{n_{2}} \\
\xi_{i}=|x| / \tau^{1 / \mu_{i}} \\
\mu_{i}=m_{i}+2 p-3, i=1,2
\end{gathered}
$$

For this we prove the following theorem: 
Theorem 2: If $E_{P_{i}}(x, \tau), \mathrm{i}=1,2$ is solution to the following system

$$
\left\{\begin{array}{l}
\frac{\partial u_{1}}{\partial \tau}=\frac{\partial}{\partial x}\left(D_{1} u_{2}^{m_{1}-1}\left|\frac{\partial u_{1}}{\partial x}\right|^{p-2} \frac{\partial u_{1}}{\partial x}\right) \\
\frac{\partial u_{2}}{\partial \tau}=\frac{\partial}{\partial x}\left(D_{2} u_{1}^{m_{2}-1}\left|\frac{\partial u_{2}}{\partial x}\right|^{p-2} \frac{\partial u_{2}}{\partial x}\right),
\end{array}\right.
$$

Then for solution of the system (1) is true the following expression

$$
\begin{array}{r}
\lim _{\tau \rightarrow \infty}\left|u_{1}(x, \tau)-E_{P_{1}}(x, \tau)\right|=0, \\
\lim _{\tau \rightarrow \infty}\left|u_{2}(x, \tau)-E_{P_{2}}(x, \tau)\right|=0
\end{array}
$$

In the set of

$$
\left\{x \in R:|x|<c \tau^{1 / \mu}, c=\max \left\{c_{1}, c_{2}\right\}>0\right\} .
$$

Proof.

Let

$$
u_{i}(x, \tau), \mathrm{i}=1,2
$$

Are solution of the following system

$$
\left\{\begin{array}{l}
\frac{\partial u_{1}}{\partial \tau}=\frac{\partial}{\partial x}\left(D_{1} u_{2}^{m_{1}-1}\left|\frac{\partial u_{1}}{\partial x}\right|^{p-2} \frac{\partial u_{1}}{\partial x}\right)-a_{1} \tau^{b_{1}} k^{\beta_{1}+3-m_{1}-2 p} u_{1}^{\beta_{1}+1} \\
\frac{\partial u_{2}}{\partial \tau}=\frac{\partial}{\partial x}\left(D_{2} u_{1}^{m_{2}-1}\left|\frac{\partial u_{2}}{\partial x}\right|^{p-2} \frac{\partial u_{2}}{\partial x}\right)-a_{2} \tau^{b_{2}} k^{\beta_{2}+3-m_{2}-2 p} u_{2}^{\beta_{2}+1}
\end{array}\right.
$$

From theorem 1 the family of solutions

$$
u_{i k}(x, \tau)=\mathrm{k} u_{i}\left(\mathrm{k} x, \mathrm{k}^{\mu_{i}} \tau\right), \mathrm{i}=1,2
$$

is a compact set, and

$$
u_{i k}(x, 1)=k u_{i}\left(k x, k^{\mu_{i}}\right)=\left[a-|x| \frac{p}{p-1}\right]^{\frac{(p-1)\left(p-\left(m_{i}+1\right)\right.}{(p-2)^{2}-\left(m_{1}-1\right)\left(m_{2}-1\right)}}=E_{P_{i}}(x, 1) . \mathrm{i}=1,2
$$


If we take $k x=x^{\prime}, k^{\mu}=\tau^{\prime}$

Then

$$
x=\frac{x^{\prime}}{k}, k=\tau^{\frac{1}{\mu}}
$$

and taking into account (10)

$\tau^{\frac{1}{\mu_{i}}} u_{i}\left(x^{\prime}, \tau^{\prime}\right)=E_{P_{i}}\left(\frac{x^{\prime}}{k}, 1\right)=\tau^{\frac{1}{\mu_{i}}} \tau^{\prime}{ }^{-\frac{1}{\mu_{i}}}\left[a-\left(\frac{k|x|}{k}\right)^{\left.\frac{p}{p-1}\right]^{\frac{(p-1)\left(p-\left(m_{i}+1\right)\right.}{(p-2)^{2}-\left(m_{1}-1\right)\left(m_{2}-1\right)}}}=\tau^{\frac{1}{\mu_{i}}} E_{P_{i}}\left(\mathrm{x}^{\prime}, \tau^{\prime}\right) \mathrm{i}=1,2\right.$.

Because $\tau^{\frac{1}{\mu_{i}}} \neq 0$ from (11)

$$
u_{i}\left(x^{\prime}, \tau^{\prime}\right)=E_{P_{i}}\left(\mathrm{x}^{\prime}, \tau^{\prime}\right) \mathrm{i}=1,2
$$

Thus, in a compact set holds following expression

$$
\begin{aligned}
& \lim _{\tau \rightarrow \infty}\left|u_{1}(x, \tau)-E_{P_{1}}(x, \tau)\right|=0, \\
& \lim _{\tau \rightarrow \infty}\left|u_{2}(x, \tau)-E_{P_{2}}(x, \tau)\right|=0 .
\end{aligned}
$$

Theorem 2 is proved.

In view of theorem 2, we define ${ }_{a}$ in the following way.

If the condition (9), and is defined as follows:

$$
a=\left(P_{1} \gamma / B\left(1 / \gamma, 1+n_{1}\right)\right)^{\frac{\gamma}{n_{1}}}=\left(P_{2} \gamma / B\left(1 / \gamma, 1+n_{2}\right)\right)^{\frac{\gamma}{n_{2}}} .
$$

Because

$$
\begin{aligned}
& \left\{\begin{array}{c}
\tau^{-\frac{1}{\mu_{1}}} \int_{-\infty}^{\infty}\left(a-\xi_{1}^{\gamma}\right)_{+}^{{ }^{n_{1}}} d x=P_{1} \\
\tau^{-\frac{1}{\mu_{2}}} \int_{-\infty}^{\infty}\left(a-\xi_{2}^{\gamma}\right)_{+}{ }^{n_{2}} d x=P_{2}
\end{array}\right. \\
& \xi_{i}=\frac{x}{\tau^{1 / \mu_{i}}},\left[\frac{x}{\tau^{1 / \mu_{i}}}\right]^{\frac{p}{p-1}}=a \eta
\end{aligned}
$$




$$
\begin{gathered}
x=a^{\frac{p-1}{p}} \tau^{\frac{1}{\mu_{i}}} \eta^{\frac{p-1}{p}} d x=a^{\frac{p-1}{p}} \tau^{\frac{1}{\mu_{i}}} \frac{p-1}{p} \eta^{\frac{p-1}{p}-1} d \eta \\
\tau^{-\frac{1}{\mu_{1}}} \int_{-\infty}^{\infty}\left(a-\xi_{1}^{\gamma}\right)_{+}^{n_{1}} d x=a^{\frac{n_{1}}{\gamma}} \frac{1}{\gamma} \int_{0}^{1} \eta^{\frac{1}{\gamma}-1}(1-\eta)^{n_{1}} d \eta=a^{\frac{n_{1}}{\gamma}} \frac{1}{\gamma} B\left(\frac{1}{\gamma}, 1+n_{1}\right)=P_{1} \\
\tau^{-\frac{1}{\mu_{2}}} \int_{-\infty}^{\infty}\left(a-\xi_{2}^{\gamma}\right)_{+}^{n_{2}} d x=a^{\frac{n_{2}}{\gamma}} \frac{1}{\gamma} \int_{0}^{1} \eta^{\frac{1}{\gamma}-1}(1-\eta)^{n_{2}} d \eta=a^{\frac{n_{2}}{\gamma}} \frac{1}{\gamma} B\left(\frac{1}{\gamma}, 1+n_{2}\right)=P_{2}
\end{gathered}
$$

Here

$$
a=\left[P_{1} \gamma / B\left(\frac{1}{\gamma}, 1+n_{1}\right)\right]^{\frac{\gamma}{n_{1}}}=\left[P_{2} \gamma / B\left(\frac{1}{\gamma}, 1+n_{2}\right)\right]^{\frac{\gamma}{n_{2}}} .
$$

Obtained computational experiments and numerical results.

\section{CONCLUSION}

Thus, the adequate study of nonlinear equations, biological populations with double-linearity based on the method of nonlinear splitting and numerical study of nonlinear processes described by equations with a double nonlinearity and analysis of the results, based on the obtained estimates of the solutions gives a comprehensive picture of the process of multicomponent systems competing biological population.

\section{REFERENCES}

[1] Aripov M. the Method of standard equations for the solution of nonlinear boundary value problems, Tashkent, Fan, 1988, 137 p.

[2] Belotelov N. In. Lobanov A. I. Population model with nonlinear diffusion. // Mathematical modeling. -M., 1997, №12, p. 43-56.

[3] V. Volterra. Mathematical theory of struggle for existence), Moscow: Nauka, 1976, 288 p.

[4] Gauze G. F. destruction of one species by another in the populations of ciliates // Zoology journal,1934, vol. 13 , No. 1.

[5] Aripov M., Muhammadiev J. Asymptotic behaviour of automodel solutions for one system of quasilinear equations of parabolic type. Buletin StiintificUniversitatea din Pitesti, Seria Matematica si Informatica. N 3. 1999. pg. 19-40

[6] Aripov M.M. Muhamediyeva D.K. To the numerical modeling of self-similar solutions of reactiondiffusion system of the one task of biological population of Kolmogorov-Fisher type. International Journal of Engineering and Technology. Vol-02, Iss11, Nov-2013. India. 2013.
[7] 7. Aripov M. M. Mukhamadieva D. K. Approaches to the solution of one task of biological population. Questions of computational and applied mathematics. -Tashkent. 2013. Vol.129. -P. 22-31.

[8] Murray George. Nonlinear diffusion equations in biology. M., Mir,1983, p. 397

[9] Huashui Zhan «The asymptotic behavior of solutions for a class of doubly degenerate nonlinear parabolic equations» (Journal of Mathematical Analysis and Applications (370) 2010).

[10] Sergey Petrovskii, Nanako Shigesada. Some exact solutions of a generalized Fisher equation related to the problem of biological invasion. Mathematical biosciences. 172 (2001), 73-94.

$$
\left\{\begin{array}{l}
\frac{\partial u}{\partial t}=\operatorname{div}\left(D_{1} v^{m_{1}-1}|\nabla u|^{p-2} \nabla u\right)+k_{1} u\left(1-u^{\beta_{1}}\right) \\
\frac{\partial v}{\partial t}=\operatorname{div}\left(D_{2} u^{m_{2}-1}|\nabla v|^{p-2} \nabla v\right)+k_{2} v\left(1-v^{\beta_{2}}\right)
\end{array}\right.
$$

1. Fast Diffusion: As initial approximation we must take:

$$
\begin{gathered}
u_{0}(x, t)=(T+t)^{-\alpha_{1}}\left(a+\xi^{\gamma}\right)^{n_{1}}, \\
v_{0}(x, t)=(T+t)^{-\alpha_{2}}\left(a+\xi^{\gamma}\right)^{n_{2}}, \\
\xi=\frac{|x|}{\tau^{\frac{1}{p}}} \gamma=\frac{p}{p-1},
\end{gathered}
$$




$$
n_{i}=\frac{(p-1)\left[p-\left(m_{i}+1\right)\right]}{q}
$$

$$
\int_{-\infty}^{\infty} u_{2}(x, 0) d x=P_{2}
$$

$$
i=1,2, \quad q=(p-2)^{2}-\left(m_{1}-1\right)\left(m_{2}-1\right)
$$

Parameter values must be $n_{1}>0, \mathrm{n}_{2}>0, q<0 \quad$ The constant a is determined from the condition

$$
a=\left(P_{1} \gamma / B\left(\frac{1}{\gamma}, 1+n_{1}\right)\right)^{\frac{\gamma}{n_{1}}}=\left(P_{2} \gamma / B\left(\frac{1}{\gamma}, 1+n_{2}\right)\right)^{\frac{\gamma}{n_{2}}},
$$

$$
\int_{-\infty}^{\infty} u_{1}(x, 0) d x=P_{1}
$$

$$
k_{1}=1 / \beta_{1}, k_{2}=1 / \beta_{2}
$$

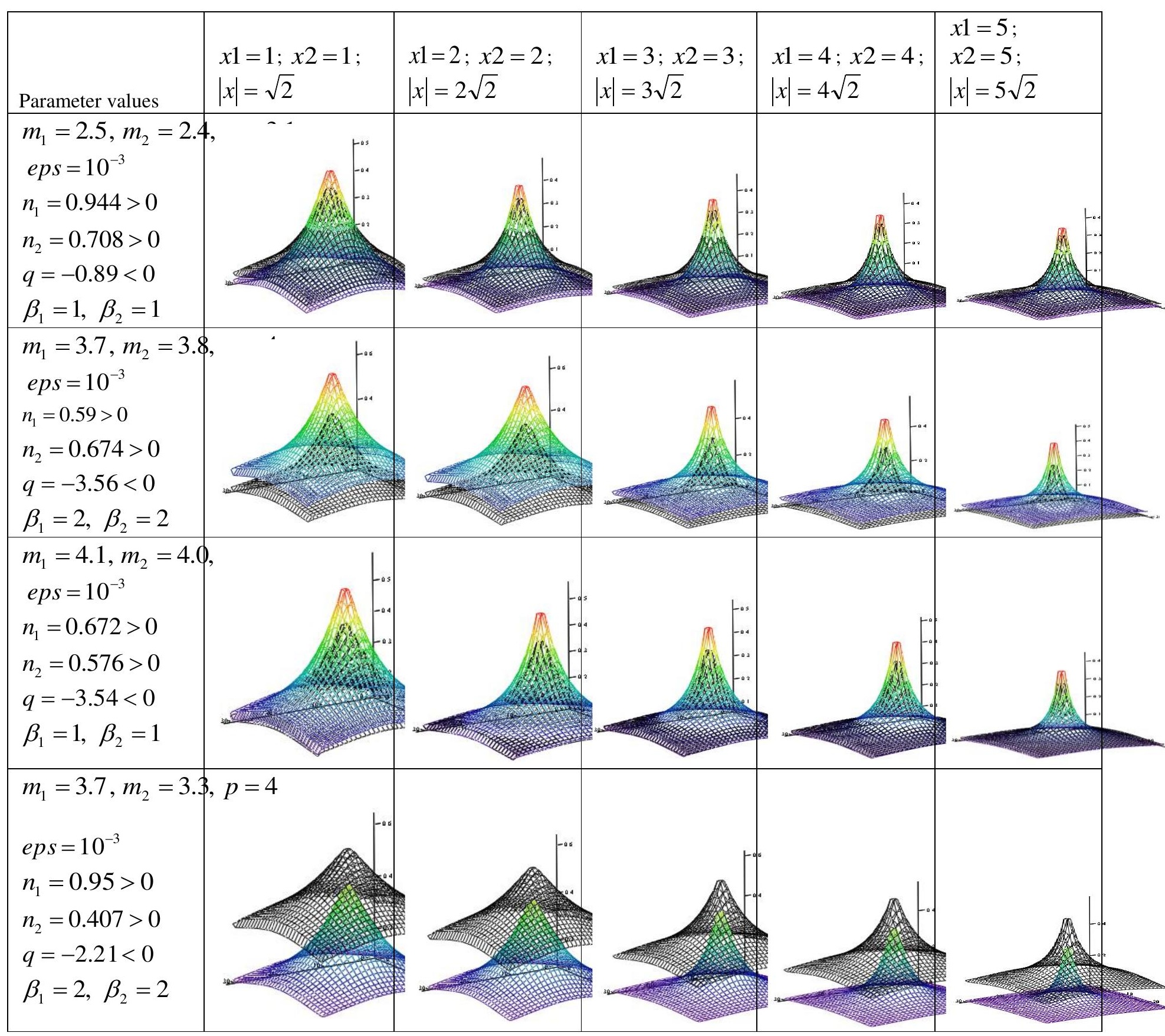




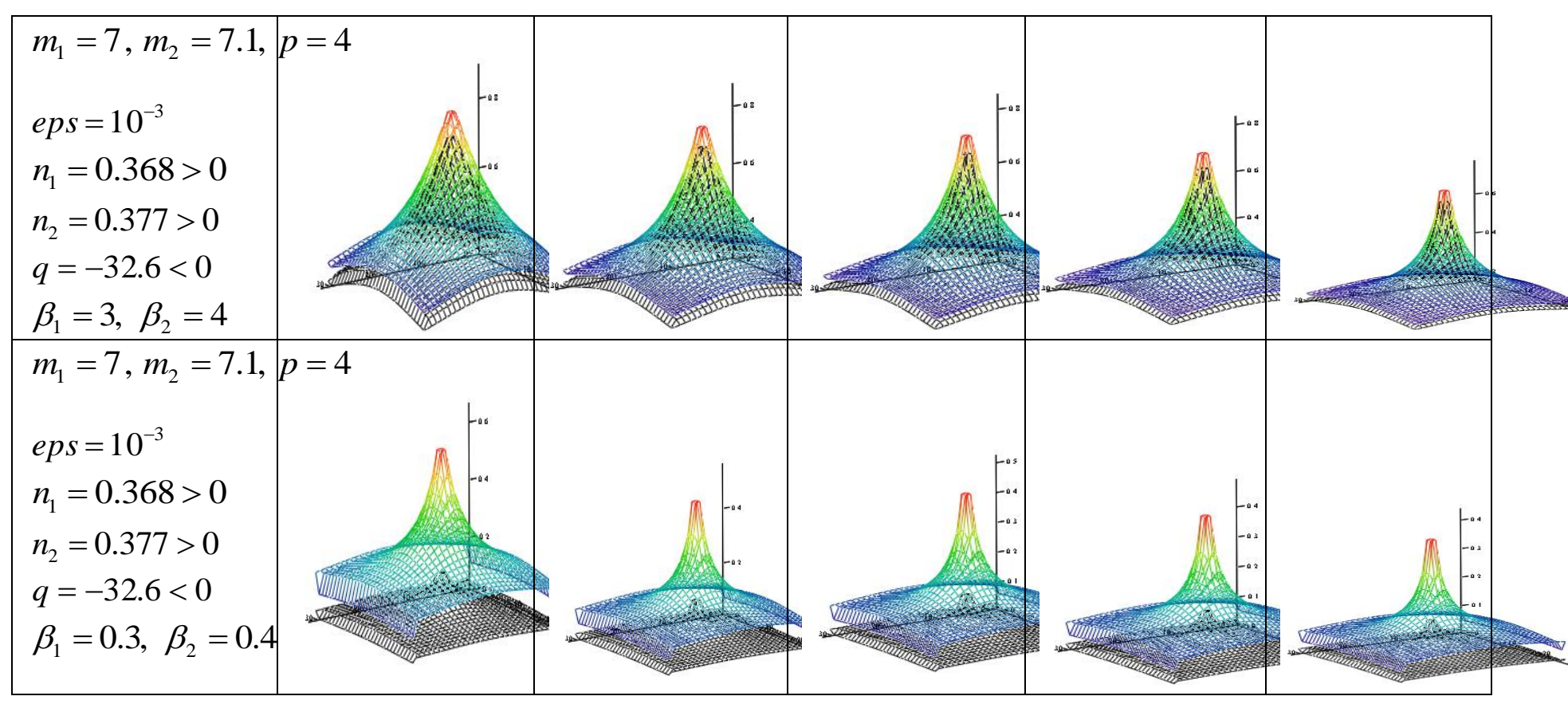

2. Slow Diffusion: As initial approximation should be taken

$$
q=(p-2)^{2}-\left(m_{1}-1\right)\left(m_{2}-1\right)
$$

$$
\begin{gathered}
u_{0}(x, t)=(T+t)^{-\alpha_{1}}\left(a-\xi^{\gamma}\right)_{+}^{n_{1}}, \\
v_{0}(x, t)=(T+t)^{-\alpha_{2}}\left(a-\xi^{\gamma}\right)_{+}^{n_{2}}, \\
\xi=\frac{|x|}{\tau^{\frac{1}{p}}} \gamma=\frac{p}{p-1}, \mathrm{\tau}, i=1,2,
\end{gathered}
$$

\begin{tabular}{|c|c|c|c|c|c|}
\hline Parameter values & $\begin{array}{l}x 1=1 ; x 2=1 ; \\
|x|=\sqrt{2}\end{array}$ & $\begin{array}{l}x 1=2 ; x 2=2 ; \\
|x|=2 \sqrt{2}\end{array}$ & $\begin{array}{l}x 1=3 ; x 2=3 ; \\
|x|=3 \sqrt{2}\end{array}$ & $\begin{array}{l}x 1=4 ; \\
x 2=4 ; \\
|x|=4 \sqrt{2}\end{array}$ & $\begin{array}{l}x 1=5 ; \\
x 2=5 ; \\
|x|=5 \sqrt{2}\end{array}$ \\
\hline $\begin{array}{l}m_{1}=1.3, m_{2}=1.2, \\
\text { eps }=10^{-3} \\
n_{1}=1.579>0 \\
n_{2}=2.368>0 \\
q=0.19>0 \\
\beta_{1}=1, \beta_{2}=1\end{array}$ & $p=2.5$ & & M & & \\
\hline $\begin{array}{l}m_{1}=1.9, m_{2}=1.7, \\
\text { eps }=10^{-3} \\
n_{1}=0.541>0 \\
n_{2}=1.622>0 \\
q=0.37>0 \\
\beta_{1}=2, \beta_{2}=2\end{array}$ & $p=3$ & 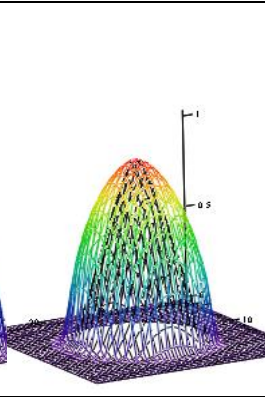 & ${ }^{17 .}{ }_{-\infty}^{-\infty}$ & & \\
\hline
\end{tabular}

Parameter values must be $n_{1}>0, n_{2}>0, q_{i}>0$, The constant $a$ is determined from the condition :

$$
\begin{aligned}
& a=\left(P_{1} \gamma / B\left(\frac{1}{\gamma}, 1+n_{1}\right)\right)^{\frac{\gamma}{n_{1}}}=\left(P_{2} \gamma / B\left(\frac{1}{\gamma}, 1+n_{2}\right)\right)^{\frac{\gamma}{n_{2}}}, \\
& k_{1}=1 / \beta_{1}, k_{2}=1 / \beta_{2}
\end{aligned}
$$




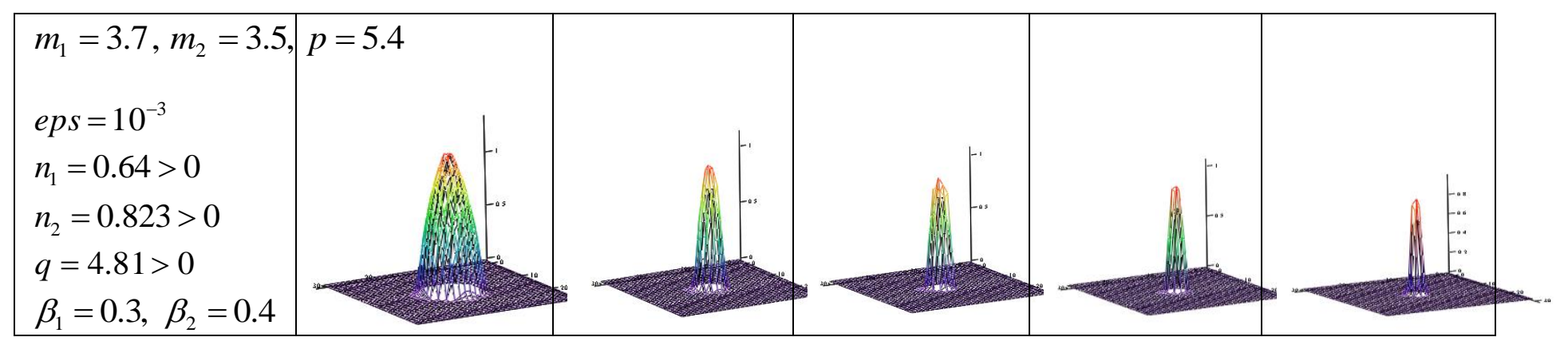

